\title{
Inferential Active Disturbance Rejection Control of a Heat Integrated Distillation Column Using Dynamic Principal Component Regression Models
}

\author{
Fahad Al-Kalbani and Jie Zhang \\ School of Chemical Engineering and Advanced Materials \\ Newcastle University \\ Newcastle Upon Tyne, NE1 7RU, UK \\ E-mail: f.k.s.al-kalbani@newcastle.ac.uk, jie.zhang@newcastle.ac.uk
}

\begin{abstract}
This paper presents a multivariable inferential active disturbance rejection control strategy for product composition control in a heat integrated distillation column (HIDiC) to overcome the large measurement delay associated with composition measurement. The inferential estimator uses multiple tray temperature measurements to estimate the product compositions. Dynamic principal component regression is used to overcome the strong co-linearity among tray temperatures and incorporate dynamics to build the inferential estimator model. The effectiveness of the proposed method is demonstrated on a simulated HIDiC based on mechanistic model.
\end{abstract}

Keywords-Distillation collumn control; heat integrated distillation column, active disturbance rejection control, inferential estimator.

\section{INTRODUCTION}

Distillation is one of the most energy consuming units in refinery and chemical industries where it is requires $40 \%$ of operational energy to operate [1]. Thus the industry needs to adhere some efforts on the development of new advanced technologies to reduce the energy consumption by using heat integration technology in distillation process. One new technology in this area is called heat integrated distillation column (HIDiC) technology [2]. The main concept of HIDiC is to distribute the exergy loss from the main reboiler and condenser to all section stages, thus reducing the overall exergy loss and the main utility load of the section. If two diabatic columns with opposite heat transfer direction are integrated, the energy demand along both columns can then be reduced and saved [3]. According to Fig.1, the stripping and rectifying sections are separated by the feed input tray. Instead of designing heat supply to pass only through the main reboiler and heat rejection only through the main condenser, the whole heat rejection is passed and circulated along the rectifying section, while the same amount of heat by the stripping section to distribute it through the column. A throttling valve and compressor are used to control the pressure difference between both column sections. The pressure in the rectifying column section must be sufficiently high in order to enable the heat transfer along the column by providing a positive temperature driving force. The heat duties of the reboiler and condenser will correspondingly be minimized or even eliminated, hence saving a large amount of energy.

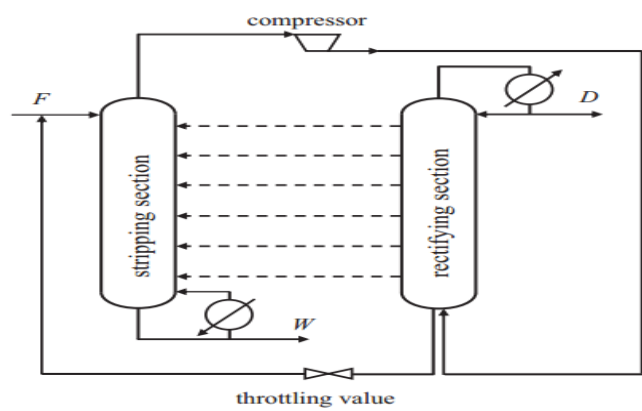

Fig. 1. The schematic diagram of HIDiC

HIDiC is also expected to produce a significant energy savings of about $30-50 \%$ in various mixture separations when compared to the conventional column [4]. Although the HIDiC has huge potentials of energy saving, the heat integration between the stripping and rectifying column sections leads to critical and complex dynamics behavior [5], such as large response lags, high steady state gains, strong magnitude and direction dependences of disturbances, and asymmetric dynamics that were addressed by Zhu and Liu [6]. Various control schemes were introduced to identify the most appropriate approach for the HIDiC [7]. Moreover, control of HIDiC for propylene - propane splitter and benzene - toluene separation has been reported recently [8]. Currently, most HIDiCs are controlled by using single loop ProportionalIntegral and Derivative control (PID), Model Predictive Control (MPC), and many other control schemes. In this paper, Active Disturbance Rejection Control (ADRC) will be implemented and integrated with the inferential control to effectively control the HIDiC scheme.

This paper is structured as follows: Section II provides an overview of active disturbance rejection control and inferential control. The integration of ADRC and the inferential control scheme is explained in Section III. In this section, the inferential model of static and dynamic PCR model are introduced in order to integrate it with the ADRC schemes. The last section draws some concluding remarks. 


\section{OVERVIEW OF ADRC AND INFERENTIAL CONTROL}

Due to the tightening of safety and environmental regulations, strong global competition, and the insatiable desire for better product quality, the chemical processes operation must continuously improve its efficiency level in order to meet those requirements. Effective control technique is the key factor in achieving this goal [5].

PID control is a popular control scheme that dates back to 1920s. Although the control hardware has already entered the digital era, PID control is still the most commonly used control approach in process control. In order to overcome the PID limitations [9-10], model based control, such as MPC had been successfully developed and applied. Plenty of linear MPC applications can be identified in different industries. Although the model based approach provides many advantages, there are various potential limitations such as the dependence of an accurate model, the huge number of state variables in the model, and the tuning and optimization process needs designer experience with trial and error tests that might be quite expensive [11-12]. As a result, ADRC was introduced and developed outside the area of process control [13-14]. It has been successfully applied in various complex control problems and it shows an excellent dynamic performance in set-point tracking and disturbance rejection. The main idea of ADRC is to use an Extended State Observer (ESO) to predict the external and internal disturbances on-line. The ADRC scheme has two important features which are as follow:

- The independence of an accurate plant model.

- The strong disturbance rejection capability.

According to Fig. 2, ADRC consists of three main components which are transient profile generator (TPG), extended state observer (ESO), and non-linear weighted sum (N-LWS) or non-linear feedback control function (NLFC). Each individual component has specific functions, which are presented below.

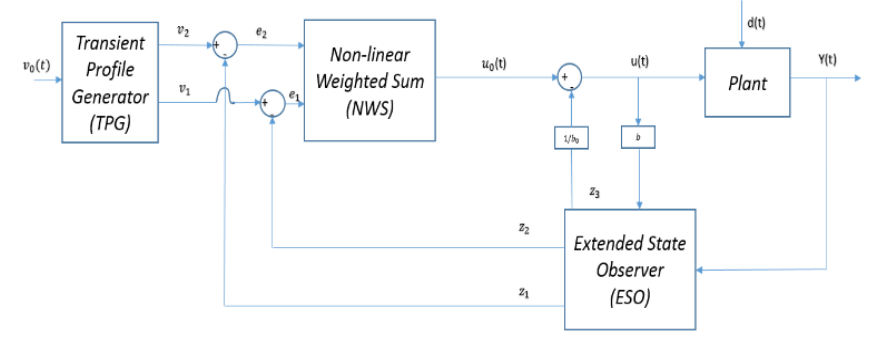

Fig.2. Structure of ADRC scheme

\section{A. Transient Profile Generator}

Since the current practical system has dynamic behaviour and it cannot reach the desired signal sufficiently fast that usually introduce large initial error and other common problems saturation. In order to overcome this type of limitation, Han introduced Transient Profile Generator (TPG) which is used to arrange the process of transition to generate smooth output signal and its differential signal from the input signal. It is also used to reduce the undesired overshoot, keep the speed of the system and guarantee the rapidity of the system response [15]. TPG can be described as [16]:

$$
\left\{\begin{aligned}
& \dot{v}_{1}=v_{2} \\
& \dot{v}_{2}=v_{3} \\
&: \\
& \dot{v}_{n-1}=v_{n} \\
& \dot{v}_{n}=\lambda^{n} \psi\left(v_{1}-\mathrm{h}, \frac{v_{2}}{\lambda}, \ldots, \frac{v_{n}}{\lambda^{n-1}}\right)
\end{aligned}\right.
$$

where $h$ indicates the input force, $v_{i}=(i=1,2, \ldots, n)$ is the output signal of TPG, and $\lambda$ is a factor of adjustable speed. This parameter can be increased and decreased based on the application [17].

\section{B. Extended State Observer}

The extended state observer (ESO) introduced by Han in [9] provides successful and efficient disturbance estimation even without having an accurate model or knowledge of the plant system. It is used to estimate exogenous and/or internal disturbances in the concern of their following cancelation [18]. Due to the generalized principle of disturbance and the strong capability of ESO, it allows the ADRC scheme to achieve the desired performance and handle large amounts of uncertainties [19]. It also pays the suitable compensation by turning the uncertain and nonlinear system containing the unknown disturbance into a linear system. Hence, the non-linear system feedback can be linearized finally to an integrator tandem approach that simplifies the controlled objects and enhances the controlling property. ESO can be designed as follows [20]:

$$
\left\{\begin{aligned}
e= & z_{1}-y \\
\dot{z}_{1}= & z_{2}-\beta_{1} \cdot \varphi_{1}(e) \\
\dot{z}_{2}= & z_{3}-\beta_{2} \cdot \varphi_{2}(e) \\
& : \\
\dot{z}_{n}= & z_{n+1}-\beta_{n} \cdot \varphi_{n}(e)+b . u \\
\dot{z}_{n+1}= & -\beta_{n+1} \cdot \varphi_{n+1}(e)
\end{aligned}\right.
$$

where $z_{i}(i=1,2, \ldots, n+1)$ are the ESO outputs which indicates state variables and real time disturbance, $\beta_{i}(i=$ $1,2, \ldots, n+1)$ indicates the gain of observer and $\varphi_{n}(e)(i=$ $1,2, \ldots, n+1)$ are the non-linear functions, mostly, described as [30]:

$$
\varphi_{n}(e)=f a l(e, \alpha, \delta)= \begin{cases}\frac{e}{\delta^{1-\alpha}} & |e| \leq \delta \\ |e|^{\alpha} \operatorname{sgn}(e) & |e|>\delta\end{cases}
$$$$
\text { where } \delta \text { and } \alpha \text { are two important variables to be }
$$
predetermined.

\section{Non-linear Weighted Sum}

Non-linear state error feedback (NLSEF) is implemented to calculate the error in the controlled variable by using the nonlinear combination of feedback state error and the outputs from the ESO and TPG. It also combines the disturbance compensation with the error variables to guarantee a good performance of the plant system [21].

The non-linear error feedback law is shown below[22]

$e_{1}=v_{1}-z_{1}$

$e_{2}=v_{2}-z_{2}$

The NLSEF control law can be implemented as: $u=\frac{u_{0}-z_{n+1}}{b_{0}}$ 
where $u_{0}$ is desired closed loop dynamic, and $b_{0}$ is a rough approximation of the input parameter $b$.

However, the control of distillation product compositions is really a challenging task because of the existence of large time delays in composition measurement introduced by current measuring devices such as gas analyzer. Hence, in the presence of undesired and unmeasured disturbances, inferential control is one of the common technical solutions that can be used to estimate an inaccessible process output. While, in most distillation columns, the product compositions are strongly related to tray temperatures [23]. In multicomponent distillation, temperature control is not always an effective solution for composition control, since the temperature profile does not represent exactly the product compositions [24]. Therefore, it is essentially important to infer the product compositions from secondary measurements like flows, pressures, temperatures, etc. The inferential estimator can be designed by using more than one temperature measurement to infer the output compositions. The resulting estimated signal can be used by the controller to work out the control actions.

There are many successful design and implementations of inferential control methods in the literature. Two of the earliest papers in this area investigated the need to estimate the difficult-to-measure primary variables with efficient, reliable and faster secondary measurements to enhance the closed loop response [25, 26]. Subsequent publications from Brosilow and co-authors discussed the development of inferential control schemes with emphasis on both static and dynamic estimation of the unmeasured primary variable [27-31].

\section{INFERENTIAL ACTIVE DISTURBANCE REJECTION CONTROL}

The designed inferential ADRC scheme for HIDiC is shown in Fig. 3 that consists of three main parts which are as follows:

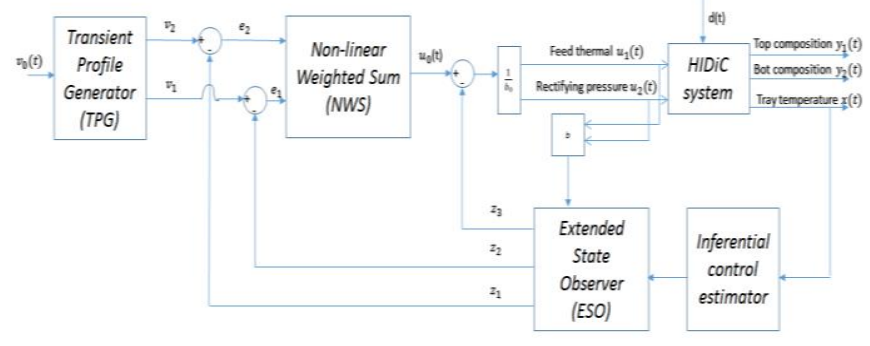

Fig. 3. Structure of inferential ADRC scheme

i. Inferential Control Estimator: In order to control the top and bottom product compositions $y_{1}$ and $y_{2}$ and due to the large measurements delay introduced by the current product composition analyzers, soft sensors are implemented to estimate the product compositions (primary controlled variables) from the secondary measurements. Since the product compositions are closely related to tray temperatures, the tray temperatures are used as the secondary measurement variables $(x)$ to estimate both product compositions online. The inferred product compositions are used as the feedback signals in the control loop. Principal component regression (PCR) is used to build the static and dynamic soft sensors.

ii. ADRC: The estimated product compositions are fed to the ADRC via the ESO component. Since the process system is usually subjected to model parametric uncertainty and various external disturbances (the external disturbance in this process is the feed composition) that may affect the overall dynamic performance of the system, the ESO will eliminate their impacts successfully before it enter the whole system by generating an estimation signals that will be compared with the TPG output signal in order to obtain the error signal which will be given to the N-LSEF and generate the suitable control signal. The ADRC controller will manipulate two manipulating variables which are feed thermal rate $(q)$ represented as $\left(\mathrm{u}_{1}(\mathrm{t})\right)$ and Rectifying Pressure $(P r)$ represented as $\left(\mathrm{u}_{2}(\mathrm{t})\right)$ respectively to reduce the difference between the reference and measured composition.

iii. Process: In this research, a generic non-linear mechanistic model of an HIDiC with 54 trays for separating Benzene (top product $y_{1}$ ) and Toluene (bottom product $y_{2}$ ) is used to simulate the process [32]. The mechanistic model is based on the following assumptions: the liquid and vapour stream leaving each tray are in equilibrium condition, the vapour and liquid on each stage are perfectly mixed with uniform temperature on each stage, the vapour holdups are negligible, constant liquid holdups in each stage, negligible heat capacity and heat loss between rectifying and stripping sections, the transportation delay of vapour and liquid between stages is negligible, equal and constant component latent heat and constant relative volatility. Table I presents the nominal operating conditions considered on this research.

TABLE I. NOMINAL OPERATING CONDITIONS

\begin{tabular}{|l|l|}
\hline \multicolumn{1}{|c|}{ Property name } & Numerical value \\
\hline Stage number & 54 trays \\
\hline Feed stage & 27 \\
\hline Feed flow rate & $83.33 \mathrm{kmol}^{-1}$ \\
\hline Top composition (Benzene) & $99.5 \%$ \\
\hline Bottom composition (Toluene) & $0.5 \%$ \\
\hline Feed thermal condition & $0-1$ \\
\hline Feed composition $\left(d_{1}\right)$ & $50 \%$ \\
\hline Pressure of stripping section $\left(P_{s}\right)$ & $0.1013 \mathrm{MPa}$ \\
\hline Pressure of rectifying section $\left(P_{r}\right)$ & $0.1013-1.013 \mathrm{MPa}^{-13}$ \\
\hline Heat transfer rate & $9803 \mathrm{~W} . \mathrm{K}^{-1}$ \\
\hline Latent heat of vaporization & $30001.1 \mathrm{KJ}^{-\mathrm{Kmol}^{-1}}$ \\
\hline Relative volatility & 2.317 \\
\hline
\end{tabular}


Fig. 4 presents the difficult to measure product compositions in the generated data. Fig. 5 shows the corresponding tray temperatures. It can be noticed the existence of strong correlation among tray temperature measurements.
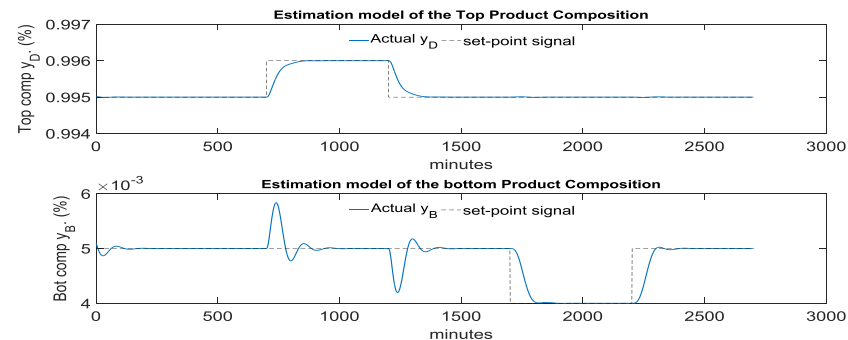

Fig. 4. Top and Bottom product compositions.

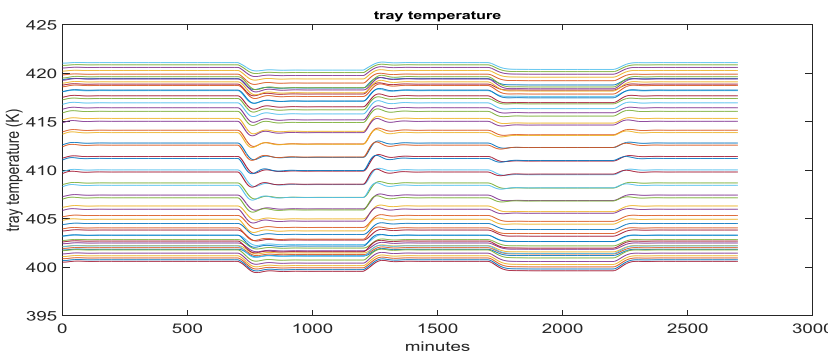

Fig. 5. Tray temperature measurements.

\section{A. Static inferential model}

The static inferential model is given below:

$y(t)=\theta_{1} T_{1}(t)+\theta_{2} T_{2}(t)+\cdots+\theta_{54} T_{54}(t)$

where $y$ represents the product compositions, $T_{1}$ to $T_{54}$ represent the temperatures from trays 1 to 54 respectively, $\theta_{1}$ to $\theta_{54}$ represent the model parameters corresponding to these tray temperatures.

Since the generated data has different ranges, the data were scaled to zero mean and unit variance in order to build PCR models. Then, the data is divided into training data with 2200 samples (samples 1 to 2200) and testing data with 2100 samples (samples 600 to 2700).

\section{B. Dynamic inferential model}

The relationship between tray temperatures and product compositions can be dynamic and, in this case, a dynamic modeling linking the secondary and primary variables is more appropriate. This dynamic soft sensors are developed by adding lagged tray temperature measurements as additional model inputs. In order to determine the order of the dynamic soft sensor, 9 dynamic inferential models have been developed with the first order model given by $\operatorname{Eq}(7)$.

$y(t)=\theta_{1.1} T_{1}(t)+\theta_{1.2} T_{2}(t-1)+\theta_{2.1} T_{1}(t)+$

$\theta_{2.2} T_{2}(t-1)+\cdots+\theta_{54.1} T_{54}(t)+\theta_{54.2} T_{54}(t-1)$

Data partition and scaling are kept the same as static PCR model. The suitable dynamic model can be determined by selecting the one with the least SSE value. Table II indicates the number of principle components with the corresponding SSE value.
Table II. NUMBER OF PC WITH THE CORRESPONDING SSE ON THE TESTING DATA OF DIFFERENT DYNAMIC PCR MODELS.

\begin{tabular}{|l|l|l|c|}
\hline $\begin{array}{c}\text { Order of dynamic } \\
\text { model }\end{array}$ & Product composition & No of PC & SSE \\
\hline \multirow{2}{*}{$1^{\text {st }}$ dynamic model } & Top Comp & 97 & $6.5880 \times 10^{-15}$ \\
\cline { 2 - 4 } & Bot comp & 92 & $1.1513 \times 10^{-12}$ \\
\hline \multirow{2}{*}{$2^{\text {nd }}$ dynamic model } & Top Comp & 74 & $2.2283 \times 10^{-14}$ \\
\cline { 2 - 4 } & Bot comp & 97 & $1.0098 \times 10^{-12}$ \\
\hline \multirow{2}{*}{$3^{\text {rd }}$ dynamic model } & Top Comp & 52 & $1.9017 \times 10^{-13}$ \\
\cline { 2 - 4 } & Bot comp & 95 & $4.6802 \times 10^{-13}$ \\
\hline \multirow{2}{*}{$4^{\text {th }}$ dynamic model } & Top Comp & 62 & $1.2760 \times 10^{-13}$ \\
\cline { 2 - 4 } & Bot comp & 61 & $5.3314 \times 10^{-13}$ \\
\hline \multirow{2}{*}{$5^{\text {th }}$ dynamic model } & Top Comp & 73 & $1.0414 \times 10^{-13}$ \\
\cline { 2 - 4 } & Bot comp & 94 & $7.8424 \times 10^{-13}$ \\
\hline \multirow{2}{*}{$6^{\text {th }}$ dynamic model } & Top Comp & 93 & $8.1893 \times 10^{-14}$ \\
\cline { 2 - 4 } & Bot comp & 26 & $1.8590 \times 10^{-12}$ \\
\hline $7^{\text {th }}$ dynamic model & Top Comp & 24 & $4.4015 \times 10^{-13}$ \\
\cline { 2 - 4 } & Bot comp & 27 & $2.4680 \times 10^{-12}$ \\
\hline $8^{\text {th }}$ dynamic model & Top Comp & 92 & $1.1390 \times 10^{-12}$ \\
\cline { 2 - 4 } & Bot comp & 27 & $3.3737 \times 10^{-12}$ \\
\hline \multirow{2}{*}{$9^{\text {th }}$ dynamic model } & Top Comp & 89 & $1.2726 \times 10^{-12}$ \\
\cline { 2 - 4 } & Bot comp & 23 & $4.4115 \times 10^{-12}$ \\
\hline
\end{tabular}

According to Tab. II, the estimation accuracy has been significantly improved compared to the static PCR model especially in the $6^{\text {th }}$ order, $7^{\text {th }}$ order and $9^{\text {th }}$ order models. Since the difference between these models is not significant, the $9^{\text {th }}$ dynamic model is used and integrated with the ADRC. Fig. 6 and Fig. 7 show, respectively, estimations from the static PCR model and the $9^{\text {th }}$ order dynamic model. In these figures, the blue solid line represents the actual compositions while the red solid line represents the corresponding estimations.

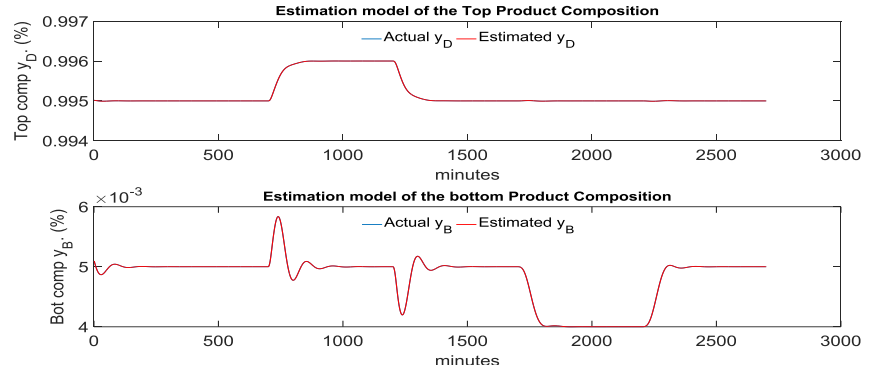

Fig. 6: Model estimation of static PCR model

Ten inferential feedback control schemes with ten different software sensors (static and the $1^{\text {st }}$ to the $9^{\text {th }}$ order dynamic PCR models) were developed. In order to analyze the performance of inferential ADRC with static and dynamic PCR models, various disturbances were introduced to the simulated HIDiC. The feed composition $z$ was increased by $15 \%$ at $300^{\text {th }}$ minutes and then decreased by $15 \%$ at $900^{\text {th }}$. Furthermore, series set-points changes are applied to both top and bottom compositions. Table III gives the SSE values of control errors with different inferential ADRC schemes. It can be realized that the dynamic inferential ADRC schemes gives better performance than the static ADRC especially those with $3^{\text {rd }}, 7^{\text {th }}$, and $9^{\text {th }}$ order dynamic soft sensors.

Fig. 8 and Fig. 9 show respectively the responses of static inferential ADRC and dynamic inferential ADRC schemes 
over a wide range of feed composition disturbances and setpoint changes. It can be seen from both figures that the setpoint signal was smoothed by ADRC scheme in order to make the output signal follow the input signal gradually which leads the efficiency of inferential ADRC scheme. Furthermore, the dynamic inferential ADRC gives better performance than the static inferential ADRC despite of large static control errors existing at bottom composition. The existence of large static error in static inferential ADRC scheme is due to the PCR model errors which might be large when the nominal operating conditions change such as set-point changes.
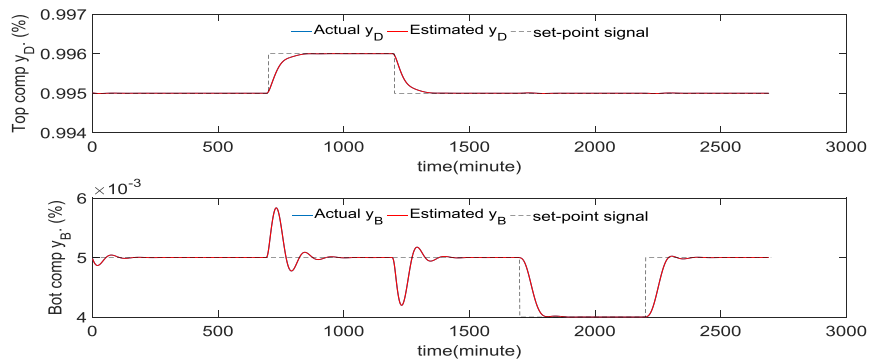

Fig. 7: Model estimation of $9^{\text {th }}$ dynamic PCR model

Table III. SSE OF DIFFERENT CONTROL SCHEME

\begin{tabular}{|c|c|c|}
\hline Order of dynamic model & $\begin{array}{c}\text { Product } \\
\text { compositions }\end{array}$ & SSE \\
\hline \multirow{2}{*}{$\begin{array}{c}\text { Static inferential ADRC } \\
\text { scheme }\end{array}$} & Top Comp & $1.5422 \times 10^{-4}$ \\
\hline & Bot comp & $2.3319 \times 10^{-5}$ \\
\hline \multirow{2}{*}{$\begin{array}{c}1^{\text {st }} \text { dynamic inferential } \\
\text { ADRC scheme }\end{array}$} & Top Comp & $1.8414 \times 10^{-5}$ \\
\hline & Bot comp & $1.4516 \times 10^{-4}$ \\
\hline \multirow{2}{*}{$\begin{array}{c}2^{\text {st }} \text { dynamic inferential } \\
\text { ADRC scheme }\end{array}$} & Top Comp & $5.6088 \times 10^{-5}$ \\
\hline & Bot comp & $2.5116 \times 10^{-4}$ \\
\hline \multirow{2}{*}{$\begin{array}{c}3^{\text {st }} \text { dynamic inferential } \\
\text { ADRC scheme }\end{array}$} & Top Comp & $8.5201 \times 10^{-6}$ \\
\hline & Bot comp & $5.0856 \times 10^{-5}$ \\
\hline \multirow{2}{*}{$\begin{array}{c}4^{\text {st }} \text { dynamic inferential } \\
\text { ADRC scheme }\end{array}$} & Top Comp & $7.0498 \times 10^{-5}$ \\
\hline & Bot comp & $1.2026 \times 10^{-5}$ \\
\hline \multirow{2}{*}{$\begin{array}{c}5^{\text {st }} \text { dynamic inferential } \\
\text { ADRC scheme }\end{array}$} & Top Comp & $2.9371 \times 10^{-5}$ \\
\hline & Bot comp & $1.0010 \times 10^{-5}$ \\
\hline \multirow{2}{*}{$\begin{array}{c}6^{\text {st }} \text { dynamic inferential } \\
\text { ADRC scheme }\end{array}$} & Top Comp & $1.7382 \times 10^{-5}$ \\
\hline & Bot comp & $1.2471 \times 10^{-5}$ \\
\hline \multirow{2}{*}{$\begin{array}{c}7^{\text {st }} \text { dynamic inferential } \\
\text { ADRC scheme }\end{array}$} & Top Comp & $6.2712 \times 10^{-5}$ \\
\hline & Bot comp & $7.6392 \times 10^{-6}$ \\
\hline \multirow{2}{*}{$\begin{array}{c}8^{\text {st }} \text { dynamic inferential } \\
\text { ADRC scheme }\end{array}$} & Top Comp & $8.9755 \times 10^{-5}$ \\
\hline & Bot comp & $8.8773 \times 10^{-5}$ \\
\hline \multirow{2}{*}{$\begin{array}{c}9^{\text {st }} \text { dynamic inferential } \\
\text { ADRC scheme }\end{array}$} & Top Comp & $7.0249 \times 10^{-6}$ \\
\hline & Bot comp & $7.8432 \times 10^{-5}$ \\
\hline
\end{tabular}

In order to eliminate the presence of static control off-sets, mean updating technique introduced by Zhang [33] is implemented. The core idea of the mean updating is that when a new steady state is reached, the new steady state values are used as the new mean values which are used in the PCR models. Thus, the model estimation will be updated. Fig. 9 and Fig. 10 show the control performance with mean updating strategy. It can be seen from both figures that static control off-sets can be successfully eliminated by using the mean updating technique.
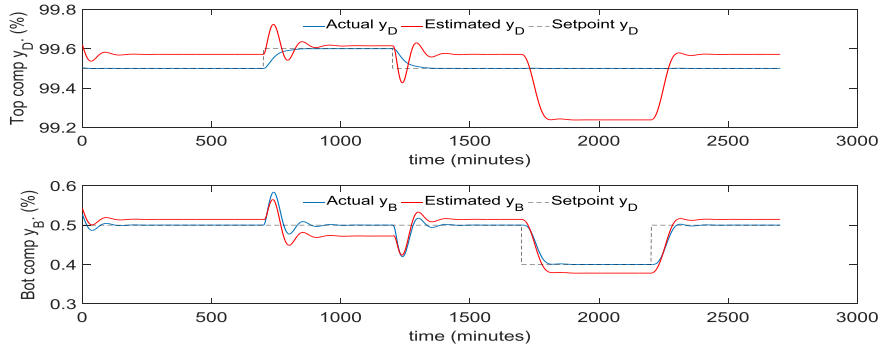

Fig. 8. Responses of actual and estimated product composition of static inferential scheme (without the mean updating technique)
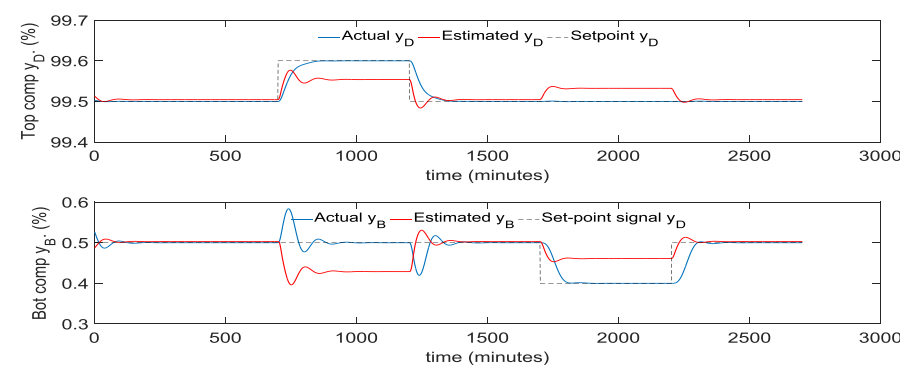

Fig. 9, Responses of actual and estimated product composition of $9^{\text {th }}$ dynamic inferential scheme (without the mean updating technique)
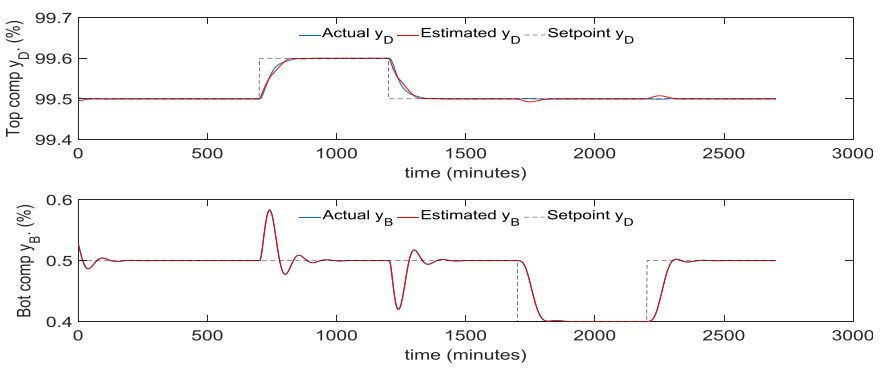

Fig. 10. Responses of actual and estimated product composition of static inferential scheme (with the mean updating technique)
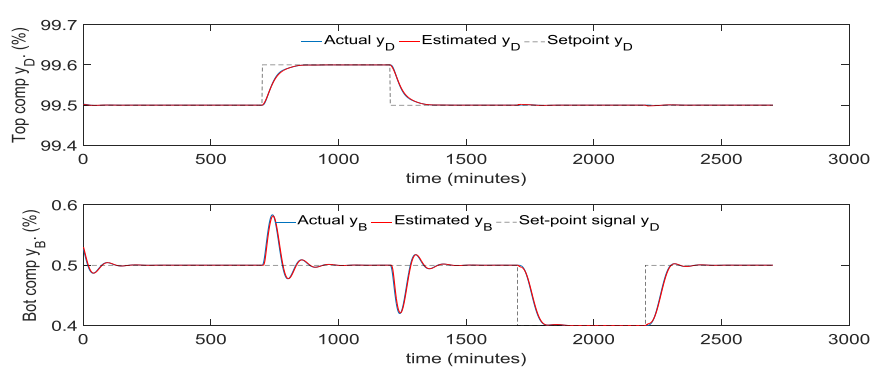

Fig. 10. Responses of actual and estimated product composition of $9^{\text {th }}$ dynamic inferential scheme (with the mean updating technique)

Table IV. SSE OF DIFFERENT CONTROL SCHEME

\begin{tabular}{|c|c|c|c|}
\hline $\begin{array}{c}\text { Control } \\
\text { schemes }\end{array}$ & Product composition & Top comp & Bot comp \\
\hline $\begin{array}{c}\text { Static } \\
\text { inferential } \\
\text { ADRC }\end{array}$ & Without mean updating & 0.4149 & 0.0092 \\
\cline { 2 - 4 } & With mean updating & $1.5422 \times 10^{-4}$ & $2.3319 \times 10^{-5}$ \\
\hline $\begin{array}{c}9^{\text {th }} \\
\text { dynamic } \\
\text { inferential } \\
\text { ADRC }\end{array}$ & Without mean updating & 0.0168 & 0.0585 \\
\cline { 2 - 4 } & With mean updating & $7.0249 \times 10^{-6}$ & $7.8432 \times 10^{-5}$ \\
\hline
\end{tabular}

Furthermore, it can be seen from Table IV that the mean updating strategy reduces the SSE value. It also proves the 
efficiency of dynamic inferential ADRC compared to the static one.

\section{CONCLUSIONS}

An inferential ADRC control scheme is proposed for HIDiC process in order to overcome the problems of large time delay associated with composition analysers. Product compositions are estimated from multiple tray temperatures using PCR. Both static and dynamic soft sensors are developed. The soft sensor outputs are fed back to ADRC. Excellent control performance is obtained with both types of soft sensors, but the dynamic PCR soft sensor gives better control performance. Moreover, the mean updating technique is used to eliminate steady state estimation bias and the resulting static control off-sets.

Due to this promising result of inferential ADRC scheme, it has been expected that will be widely implemented especially in chemical process industries to achieve higher product composition qualities at lower operating costs.

\section{ACKNOWLEDGEMENT}

The author wish to thank Thomas Bisgaard and Jakob Kjøbsted Huusom from DTU for their help in providing the simulation of HIDiC. This work is supported by Petroleum Development of Oman (PDO) and the EU FP7 (Ref: PIRSES-GA-2013612230).

\section{REFERENCES}

[1] KISS, A. A. 2014. Distillation technology-still young and full of breakthrough opportunities. Journal of Chemical Technology and Biotechnology, 89, 479-498.

[2] M. Nakaiwa, K. Huang, K. Naito, A. Endo, M. Owa, T. Akiya, 2000 "A new configuration of ideal heat integrated distillation columns (HIDiC)," Computers \& Chemical Engineering, vol. 24, pp. 239-245,

[3] K. Huang, L. Shan, Q. Zhu, and J. Qian, "Design and control of an ideal heat-integrated distillation column (ideal HIDiC) system separating a close-boiling ternary mixture," Energy, vol. 32, pp. 21482156, 2007.

[4] K. Iwakabe, M. Nakaiwa, K. Huang, T. Nakanishi, A. Røsjorde, T. Ohmori, et al., "Energy saving in multicomponent separation using an internally heat-integrated distillation column (HIDiC)," Applied thermal engineering, vol. 26, pp. 1362-1368, 2006.

[5] K. Naito, M. Nakaiwa, K. Huang, A. Endo, K. Aso, T. Nakanishi, et al., "Operation of a bench-scale ideal heat integrated distillation column (HIDiC): an experimental study," Computers \& Chemical Engineering, vol. 24, pp. 495-499, 2000.

[6] Y. Zhu and X. Liu, "Dynamics and control of high purity heat integrated distillation columns," Industrial \& engineering chemistry research, vol. 44, pp. 8806-8814, 2005.

[7] M. Nakaiwa, K. Huang, M. Owa, T. Akiya, T. Nakane, and T. Takamatsu, "Operating an ideal heat integrated distillation column with different control algorithms," Computers \& chemical engineering, vol. 22, pp. S389-S393, 1998

[8] T.-J. Ho, C.-T. Huang, J.-M. Lin, and L.-S. Lee, "Dynamic simulation for internally heat-integrated distillation columns (HIDiC) for propylene-propane system," Computers \& Chemical Engineering, vol. 33, pp. 1187-1201, 2009.

[9] J. Han, "From PID to active disturbance rejection control," IEEE transactions on Industrial Electronics, vol. 56, pp. 900-906, 2009.

[10] Z. Chen, Q. Zheng, and Z. Gao, "Active disturbance rejection control of chemical processes," in 2007 IEEE International Conference on Control Applications, 2007, pp. 855-861.

[11] M. Pizzocaro, D. Calonico, C. Calosso, C. Clivati, G. A. Costanzo, F. Levi, et al., "Active Disturbance Rejection Control: Application to the temperature stabilization of ultra-stable cavities," in European Frequency and Time Forum (EFTF), 2012, 2012, pp. 169-173.

[12] J. Han, "Auto-disturbance rejection control and its applications," Control and decision, vol. 13, pp. 19-23, 1998.

[13] J. Han, "Nonlinear design methods for control systems," in Proc. of the 14th IFAC World Congress, 1999, pp. 521-526.

[14] Z. Gao, Y. Huang, and J. Han, "An alternative paradigm for control system design," in Decision and Control, 2001. Proceedings of the 40th IEEE Conference on, 2001, pp. 4578-4585.

[15] F. Chen, H. Xiong, and J. Fu, "The control and simulation for the ADRC of USV," in Chinese Automation Congress (CAC), 2015, 2015, pp. 416-421.

[16] J. Li, Y. Xia, X. Qi, Z. Gao, K. Chang, and F. Pu, "Absolute stability analysis of non-linear active disturbance rejection control for singleinput-single-output systems via the circle criterion method," Control Theory \& Applications, IET, vol. 9, pp. 2320-2329, 2015.

[17] F. Al Kalbani and J. Zhang, "Inferential active disturbance rejection control of a distillation column using dynamic principal component regression models," in Informatics in Control, Automation and Robotics (ICINCO), 12th International Conference, 2015, pp. 358-364.

[18] H. Sira-Ramírez, Z. Gao, and L. Cuevas-Ramírez, "Tracking in interconnected gantry crane systems: A decentralized Active Disturbance Rejection Control," in Control Conference (CCC), 2014 33rd Chinese, 2014, pp. 4342-4347.

[19] W. Xue, W. Bai, S. Yang, K. Song, Y. Huang, and H. Xie, "ADRC With Adaptive Extended State Observer and its Application to AirFuel Ratio Control in Gasoline Engines," IEEE Transactions on Industrial Electronics, vol. 62, pp. 5847-5857, 2015.

[20] J. Li, X. Qi, Y. Xia, D. Ma, S. Li, and Y. Xu, "On the absolute stability of nonlinear ADRC for SISO systems," in Control Conference (CCC), 2015 34th Chinese, 2015, pp. 1571-1576.

[21] X. Gong, Q. Liu, Y. Hu, and H. Chen, "Idle speed controller design for SI engine based on ADRC," in Control Applications (CCA), 2012 IEEE International Conference on, 2012, pp. 376-381.

[22] Y. Hu, Q. Liu, B. Gao, and H. Chen, "ADRC based clutch slip control for automatic transmission," in 2011 Chinese Control and Decision Conference (CCDC), 2011, pp. 2725-2730.

[23] M. Kano, K. Miyazaki, S. Hasebe, and I. Hashimoto, "Inferential control system of distillation compositions using dynamic partial least squares regression," Journal of Process Control, vol. 10, pp. 157-166, 2000.

[24] M. Shahbazian, H. Jazayerirad, and M. Ebnali, "ANFIS Based Identification and Control of Distillation Process," Journal of Automation and Control, vol. 2, pp. 49-56, 2014.

[25] M. T. Tham, G. A. Montague, A. J. Morris, and P. A. Lant, "Softsensors for process estimation and inferential control," Journal of Process Control, vol. 1, pp. 3-14, 1991.

[26] F. J. Doyle, "Nonlinear inferential control for process applications," Journal of Process Control, vol. 8, pp. 339-353, 1998.

[27] B. Joseph and C. B. Brosilow, "Inferential control of processes: Part I. steady state analysis and design," AIChE Journal, vol. 24, pp. 485-492, 1978.

[28] C. B. Brosilow, "The structure and design of Smith predictors from the viewpoint of inferential control," in Joint Automatic Control Conference, 1979, p. 288.

[29] J. R. Parrish and C. B. Brosilow, "Inferential control applications," Automatica, vol. 21, pp. 527-538, 1985.

[30] C. Brosilow and M. Tong, "Inferential control of processes: Part II. The structure and dynamics of inferential control systems," AIChE Journal, vol. 24, pp. 492-500, 1978.

[31] J. R. Parrish and C. B. Brosilow, "Nonlinear inferential control," AIChE journal, vol. 34, pp. 633-644, 1988.

[32] T. Bisgaard, J. Kjøbsted Huusom and J. Abildskov, "A Modeling Framework for Conventional and Heat Integrated Distillation Columns", IFAC Proceedings Volumes, vol. 46, pp. 373-378, 2013.

[33] J. Zhang, "Offset-free inferential feedback control of distillation compositions based on PCR and PLS models", Chemical Engineering \& Technology, vol. 29, no. 5 , pp. 560-566, 2006. 